\title{
Impact of musculoskeletal pain on health-related quality of life among fishing sector workers
}

\author{
Beatriz Rodríguez-Romero, Salvador Pita-Fernández, Sonia Pértega-Díaz
}

\begin{abstract}
This study aims to determine the impact of musculoskeletal pain (in terms of intensity of the pain, location and functional disability due to back pain) and other factors (socio-demographic, lifestyle and co-morbidity) on the health-related quality of life on a group of shellfish gatherers. This observational transversal study included 929 shellfish gatherers (18-69 years, $98.7 \%$ women) who completed a self-administered questionnaire, including sociodemographic and lifestyle questions, co-morbidity, intensity and location of musculoskeletal pain, and Roland-Morris Disability Questionnaire (RMDQ). Health-related quality of life was assessed using the 36-item Short Form Survey (SF-36). Physical component summary (PCS) and mental component summary (MCS) of the SF-36 were considered as outcome variables. The impact of the different factors on the PCS and MCS scores was evaluated using a stepwise linear regression analysis. Physical health was found to be independently associated to intensity of musculoskeletal pain (regression coefficient, $B=-0.96)$, number of locations with musculoskeletal pain (MSP) $(B=-0.77)$, presence of pain in the hip-knee $(B=-2.26)$, self-reported rheumatic disorders $(B=-2.79)$, lower back pain $(B=-1.62)$ and age $(B=-0.06)$. Mental health was associated with the presence of self-reported depressive syndrome $(B=-1043.1)$ and RMDQ score $(B=-42.2)$. The sample had significantly lower values than the reference population in all of the dimensions of the SF-36. Intensity of the pain, pain in the hip-knee, lower back pain, functional disability due to back pain and number of locations with musculoskeletal pain were found to have a detrimental impact on the physical health of the workers. Depressive syndrome and greater functional disability due to back pain, in turn, predict worse mental health.
\end{abstract}

Keywords

Disability evaluation Health status Musculoskeletal diseases Quality of life

\section{Introduction}

Musculoskeletal disease is one of the most important problems of public health, and it has been recognised that it should be analysed from a biopsychosocial perspective [1]. The 36-item Short Form Survey (SF-36) is one of the most widely recommended generic scales for measuring the impact of musculoskeletal pain (MSP) on the physical, mental and social domains of the individual [2].

The activities that form a part of the fishing sector include shellfish gathering. In Galicia (a region in the north-west of Spain), shellfish gatherers working on foot are mostly women with a high physical workload involving, in particular, forced postures, manual handling of loads and repetitive movements. It has been demonstrated that they have a very high prevalence of MSP and an important coexistence of MSP in different anatomical regions [3].

Numerous studies have demonstrated the negative impact of MSP on the health-related quality of life (HRQL) [4-8]. In general, these patients have a lower HRQL than the reference population, and it has further been shown that the impact of musculoskeletal conditions on physical health was similar to or greater than that of the other common chronic conditions [4]. Many of these studies have been carried out on patients, but nevertheless, slightly less is known about the impact of MSP on the HRQL of the working population, especially in the fishing sector $[9,10]$.

Furthermore, other features such as sex and age [4, 5, 11, 12], co-morbidity [4, 5, 11] or factors associated with lifestyle [5,9] have also been studied in connection with HRQL, although we only possess inconclusive data on this relationship.

The aim of the study was to verify the relationship between HRQL (through the physical and mental summary components of the SF-36) and musculoskeletal pain, socio-demographic factors, lifestyle and co-morbidity in workers in the fishing sector. 


\section{Materials and methods}

This is a cross-sectional survey of workers in the fishing sector in Galicia (in the north-west of Spain). The dependent variable was health-related quality of life. Potential variables associated with HRQL included demographic factors, co-morbidity, characteristics of MSP and functional disability related to lumbar pain. The study data were gathered between December 2007 and February 2009. Informed written consent was obtained from the subjects, and the study was approved by the ethical review board (ERB) of the Autonomous Region of Galicia (Spain) (CEIC, ID number 2009/298).

\section{Participants}

The setting was all of the fishermen's associations in Galicia (a total of 40) which have members who carry out shellfish gathering on foot. This collective represents a total of 3,970 workers, $93.95 \%$ of whom are women. We studied 929 subjects, $23.4 \%$ of the total of the eligible sample (3,970 workers). This sample size made it possible to study any percentage with a certainty of $95 \%(\alpha=0.05)$ and precision of $\pm 3.2 \%$. The inclusion criteria were defined as working as a shellfish gatherer on foot who voluntarily participate in a number of workshops of preventive physiotherapy and obtaining the informed consent from the worker. A workshop was organised in each gathering area by the Galician fishing promotion agents. These agents provided materials to the workers in each association, and they agreed on the date, time and place when and where the workshops would be held in order to guarantee the greatest possible attendance. A maximum of 20 people took part in each session. Furthermore, in order to advertise these workshops, this information was included in the "International Conference on Prevention and Safety Measures in Shallow-water Fishing" and in the technical session of the "European Musculoskeletal Disorders Week" too. Both events were held in Galicia and included participation by the presidents of the women's shellfish gatherers associations.

\section{Measures}

HRQL was measured using the SF-36 [13]. We used the Spanish standard version 2, which is currently recommended [14]. This consists of 36 items that assess the state of health in eight dimensions: physical function (PF), role-physical (RP), bodily pain (BP), general health (GH), vitality (VT), social function (SF), role-emotional (RE) and mental health $(\mathrm{MH})$. These dimensions may be reduced to two scores: physical component summary (PCS) and mental component summary (MCS). The standardised scores were calculated with the values for the reference population standards (with Spanish population standards [14] from version 1 of the SF-36 for six dimensions and American population standards [13] from version 2 of the SF-36 for the two role dimensions and two summary components). A score of 50 $(\mathrm{SD}=10)$ represented the mean for the general population, and a score of 10 was $1 \mathrm{SD}$. Lower scores indicate poorer HRQL. The psychometric characteristics of the SF-36 have been studied extensively and have been found to be reliable, valid and sensitive [13].

The workers were asked to detail their socio-demographic characteristics and answer questions on their lifestyle (smoking, physical activity during leisure time, minimum $30 \mathrm{~min} /$ three times per week) and on co-morbidity. Co-morbidity was ascertained by six dichotomized questions about rheumatic disorders, depressive syndrome, diabetes, neoplasms, back surgery and other conditions.

Musculoskeletal pain was assessed by means of the following questions: Where do you regularly have pain? The options included multiple answers relating to 11 different body regions. To obtain an overall picture of concurrent MSP in the whole body, the original 11 anatomical sites were later combined to make up five larger anatomical areas [15]: neck, shoulder or higher part of the back; lower part of the back; elbow or wrist/hand; hip or knee; and leg or ankle/foot. A total score of the number of painful sites was also calculated. If the participants experienced pain in any of the regions, they were asked to specify the intensity on the verbal numerical scale (VNS). For the VNS, the worker was asked to give a score for their pain between 0 , representing "no pain", and 10, representing "the worst pain imaginable".

The functional disability related to lumbar pain was measured using the Roland-Morris Disability Questionnaire (RMDQ). This is one of the most highly recommended and commonly used scales both in clinical and research spheres. It is also the preferred instrument for use in persons with mild to moderate lower back pain. It is a quick, simple and intuitive questionnaire that can be completed individually. The score ranges from 0 (no functional disability) to 24 (severe functional disability) points. The higher the score, the greater is the degree of functional disability. The measure offers excellent reliability, validity and responsiveness [6]. 
The paper-based questionnaire used to gather data was given to the participants at the end of the workshop. Although it was self-administered, it was filled in under the supervision of a physiotherapist, who explained the aim of the study, read each of the questions out loud, answered any questions without influencing the answers, and checked the questionnaire once it was handed back in.

\section{Statistical analysis}

Descriptive analysis was performed for all variables. Quantitative variables were expressed as mean and SD, and the results for the categorical variables were expressed as percentages and $95 \%$ confidence intervals. Standardised scores for the eight SF-36 dimensions and their two summary indexes were compared to the population norms with one-sample $t$ tests. To identify those factors associated with health status, the SF-36 physical and mental component summary scores were considered as outcome variables. The interpretation of the summaries is more direct, offers greater precision, and is less complex than interpreting each of the eight dimensions [13]. Means were compared using unpaired $t$ tests, ANOVA or Mann-Whitney and Kruskal-Wallis tests. To evaluate the normality of the variables, the Kolmogorov-Smirnov test was used. Spearman's rho correlation coefficient was used to analyse the correlation among quantitative variables. To determine those MSP locations with a higher probability of poor PCS and MCS, odds ratios for summary scores < median were also calculated, with their $95 \%$ confidence interval. Stepwise multiple linear regression models were used to determine those variables independently associated with PCS and MCS scores. Those variables significantly associated with PCS and MCS scores in the univariate analysis were initially selected to be included in the multivariate model. As the MCS does not have a normal distribution, a square transformation was used before the multiple regression analysis. Two-sided tests were used, and an alpha level of $p<0.05$ was considered to be statistically significant. Analyses were performed using SPSS version 19.0 (SPSS, Inc., Chicago, IL).

\section{Results}

The sample consisted of 929 subjects (98.7\% women). The subjects' characteristics are described in Table 1. The mean age of the workers was 50.6 years $(\mathrm{SD}=8.8)$. The highest percentages of participants were women aged between 55 and $64(39.4 \%)$ and between 45 and $54(35.4 \%)$. The characteristics of the participants are similar to those who did not take part in the study (data not shown). The most frequently reported co-morbidities were rheumatic disorders $(17.2 \%)$ and depressive syndrome $(16.1 \%)$. The $66.5 \%$ of the subjects had MSP on the day of the interview, with a mean pain intensity of 6.1 $(\mathrm{SD}=1.8)$. Cervical pain $(70.9 \%)$, lumbar pain $(65.5 \%)$ and shoulder pain $(45.8 \%)$ were the most frequent. The median of pain site localizations was three sites. By grouped anatomical regions, the most common location for pain was the cervical-dorsal-shoulder region, accounting for $82.4 \%$ of the total. The mean Roland-Morris disability score was 4.9 points $(\mathrm{SD}=4.7)$. 
Table 1. Descriptive characteristics of the study population

\begin{tabular}{|c|c|c|c|}
\hline Variable & $n($ total $=929)$ & $\%(95 \% \mathrm{CI})$ & Mean \pm SD \\
\hline \multicolumn{4}{|l|}{ Socio-demographic variables } \\
\hline Age (years) & 926 & & $50.6 \pm 8.8$ \\
\hline \multicolumn{4}{|l|}{ Gender } \\
\hline Females & 917 & $98.7(97.7 ; 99.3)$ & \\
\hline Males & 12 & $1.3(0.7 ; 2.3)$ & \\
\hline Years working as shellfish gatherers & 918 & & $21.8 \pm 13.0$ \\
\hline \multicolumn{4}{|l|}{ Lifestyle characteristics } \\
\hline Smoking (yes) & 160 & $17.3(14.9 ; 19.9)$ & \\
\hline Cigarettes/day (number) & 150 & & $12.5 \pm 8.1$ \\
\hline Physical activity during leisure time (yes) & 420 & $45.3(42.1 ; 48.6)$ & \\
\hline \multicolumn{4}{|l|}{ Co-morbidity (self-report) } \\
\hline Rheumatic disorders (yes) & 159 & $17.2(14.8 ; 19.8)$ & \\
\hline Depressive syndrome (yes) & 149 & $16.1(13.8 ; 18.6)$ & \\
\hline Diabetes (yes) & 33 & $3.6(2.5 ; 5.0)$ & \\
\hline Neoplasms (yes) & 26 & $2.8(1.9 ; 4.1)$ & \\
\hline Back surgery (yes) & 9 & $1.0(0.5 ; 1.9)$ & \\
\hline Other diseases (yes) & 276 & $29.8(26.9 ; 32.8)$ & \\
\hline \multicolumn{4}{|c|}{ MSP localization according to anatomical area groupings } \\
\hline Neck/shoulders/higher back & 764 & $82.4(79.8 ; 84.8)$ & \\
\hline Lower back & 607 & $65.5(62.3 ; 68.5)$ & \\
\hline Elbow/wrist/hand & 473 & $51.0(47.8 ; 54.3)$ & \\
\hline Hip/knee & 449 & $48.4(45.2 ; 51.7)$ & \\
\hline Leg/ankle/foot & 318 & $34.3(31.3 ; 37.5)$ & \\
\hline
\end{tabular}

MSP musculoskeletal pain

The standardised score for the SF-36 for this group of workers is shown in Table 2. The sample has a significantly lower score than the population standards in all dimensions and in the two summary components. The dimensions that are most affected (worse state of health) are BP, VT and GH, and the least affected dimensions are RE and MH. The PCS was nearly 8 points below the population standards, while in the MCS, the difference was only 1.5 points.

Table 2. Standardised scores of the SF-36 health-related quality of life scales

\begin{tabular}{lrrr}
\hline Variable & Number & Mean \pm SD & Minimum-maximum \\
\hline & & & \\
Physical function $^{\mathrm{a}}$ & 925 & $45.2 \pm 8.7^{* *}$ & $16.8-56.4$ \\
Role-physical $^{\mathrm{b}}$ & 924 & $44.2 \pm 9.8^{* *}$ & $17.7-56.9$ \\
Bodily pain $^{\mathrm{a}}$ & 926 & $40.6 \pm 8.9^{* *}$ & $21.7-57.5$ \\
General health $^{\mathrm{a}}$ & 926 & $43.3 \pm 8.7^{* *}$ & $21.6-64.2$ \\
Vitality $^{\mathrm{a}}$ & 926 & $42.9 \pm 9.2^{* *}$ & $19.7-65.0$ \\
Social function $^{\mathrm{a}}$ & 927 & $44.9 \pm 11.4^{* *}$ & $5.0-55.0$ \\
Role-emotional $^{\mathrm{b}}$ & 925 & $47.9 \pm 10.1^{* *}$ & $9.2-55.9$ \\
Mental health $^{\mathrm{a}}$ & 926 & $47.4 \pm 10.5^{* *}$ & $13.5-63.3$ \\
Physical component summary $^{\mathrm{b}}$ & 924 & $42.7 \pm 9.2^{* *}$ & $14.4-64.2$ \\
Mental component summary $^{\mathrm{b}}$ & 924 & $48.5 \pm 10.9^{* *}$ & $1.9-70.6$ \\
& & & \\
\hline
\end{tabular}

$* * p \leq 0.001$, significant differences with the population mean

${ }^{a}$ The sample score is compared with Spanish population standards from version 1 of the SF-36 for six dimensions [14]

${ }^{\mathrm{b}}$ The sample score is compared with American population standards from version 2 of the SF-36 for two dimensions and components summary [13] 


\section{Factors associated with health-related quality of life}

In the bivariate analysis, those who presented rheumatic disorders had significantly lower values in the PCS summary score. The presence of MSP in any of the anatomical areas studied has also a negative and significant impact on the physical dimension of the HRQL (Table 3, Fig. 1). As shown in Fig. 1, the OR associated to a PCS score (less than median) was higher in those cases that presented hip-knee pain and leg-ankle-foot pain.

Table 3. Mean scores for the physical summary component and mental summary component according to socio-demographic variables, lifestyle, co-morbidity and location of musculoskeletal pain

\begin{tabular}{|c|c|c|c|c|c|c|}
\hline \multirow{2}{*}{ Variable } & \multicolumn{3}{|c|}{ PCS } & \multicolumn{3}{|c|}{ MCS } \\
\hline & $n$ & Mean $\pm \mathrm{SD}$ & $p$ & $n$ & Mean \pm SD & $p$ \\
\hline Gender & & & 0.124 & & & 0.441 \\
\hline Females & 912 & $42.7 \pm 9.2$ & & 912 & $48.5 \pm 10.9$ & \\
\hline Males & 12 & $46.8 \pm 8.3$ & & 12 & $50.5 \pm 12.5$ & \\
\hline Smoking & & & 0.184 & & & 0.181 \\
\hline Yes & 159 & $43.6 \pm 8.8$ & & 159 & $49.6 \pm 10.3$ & \\
\hline No & 765 & $42.6 \pm 9.2$ & & 765 & $48.2 \pm 11.1$ & \\
\hline Physical activity during leisure time & & & 0.189 & & & 0.296 \\
\hline Yes & 419 & $43.2 \pm 9.0$ & & 419 & $48.0 \pm 11.2$ & \\
\hline No & 505 & $42.4 \pm 9.3$ & & 505 & $48.9 \pm 10.7$ & \\
\hline Rheumatic disorders & & & $\leq 0.001$ & & & $\leq 0.001$ \\
\hline Yes & 159 & $37.0 \pm 8.5$ & & 159 & $46.0 \pm 11.4$ & \\
\hline No & 765 & $43.9 \pm 8.9$ & & 765 & $49.0 \pm 10.8$ & \\
\hline Depressive syndrome & & & 0.064 & & & $\leq 0.001$ \\
\hline Yes & 148 & $41.5 \pm 9.5$ & & 148 & $36.9 \pm 11.7$ & \\
\hline No & 776 & $43.0 \pm 9.1$ & & 776 & $50.7 \pm 9.3$ & \\
\hline Diabetes & & & 0.289 & & & 0.333 \\
\hline Yes & 33 & $41.1 \pm 10.8$ & & 33 & $47.0 \pm 10.8$ & \\
\hline No & 891 & $42.8 \pm 9.1$ & & 891 & $48.5 \pm 11.0$ & \\
\hline \multicolumn{7}{|l|}{ MSP localization } \\
\hline Neck/shoulders/higher back & & & $\leq 0.001$ & & & 0.001 \\
\hline Yes & 762 & $42.0 \pm 9.0$ & & 762 & $48.0 \pm 11.0$ & \\
\hline No & 162 & $46.0 \pm 9.1$ & & 162 & $50.7 \pm 10.4$ & \\
\hline Lower back & & & $\leq 0.001$ & & & 0.088 \\
\hline Yes & 606 & $41.2 \pm 9.2$ & & 606 & $48.1 \pm 10.8$ & \\
\hline No & 318 & $45.7 \pm 8.4$ & & 318 & $49.1 \pm 11.3$ & \\
\hline Elbow/wrist/hand & & & $\leq 0.001$ & & & 0.072 \\
\hline Yes & 470 & $41.0 \pm 9.1$ & & 470 & $47.9 \pm 11.0$ & \\
\hline No & 454 & $44.5 \pm 8.9$ & & 454 & $49.0 \pm 10.8$ & \\
\hline Hip/knee & & & $\leq 0.001$ & & & $\leq 0.001$ \\
\hline Yes & 447 & $39.3 \pm 8.7$ & & 447 & $47.1 \pm 11.6$ & \\
\hline No & 477 & $46.0 \pm 8.4$ & & 477 & $49.8 \pm 10.2$ & \\
\hline Leg/ankle/foot & & & $\leq 0.001$ & & & 0.003 \\
\hline Yes & 317 & $39.0 \pm 8.8$ & & 317 & $46.9 \pm 11.6$ & \\
\hline No & 607 & $44.7 \pm 8.8$ & & 607 & $49.3 \pm 10.5$ & \\
\hline
\end{tabular}

PCS physical component summary, MCS mental component summary, MSP musculoskeletal pain 

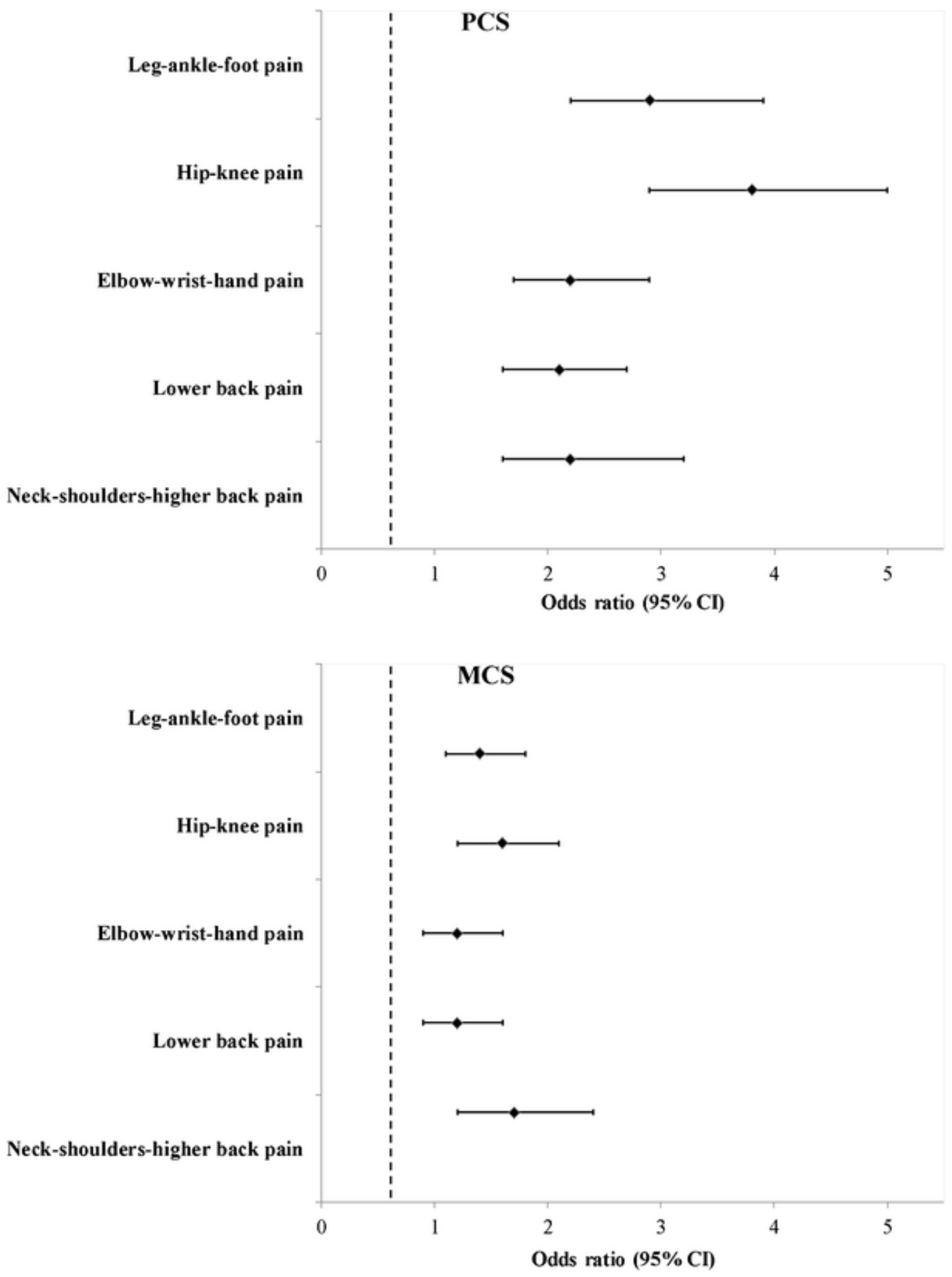

Fig. 1. Univariate association between the physical component summary $(P C S)$ and mental component summary $(M C S)(\geq$ median vs. <median) and the location of the MSP in terms of the odds ratio 
In turn, the PCS is correlated negatively and significantly with age, the amount of time spent working as a shellfish gatherer and with certain features of the MSP such as the intensity of the pain (Spearman's rho $=-0.51$ ) and the number of locations with MSP (Spearman's rho $=-0.48$ ) (Table 4). Those who referred to rheumatic disorders and depressive syndrome have significantly lower values in the MCS. The presence of MSP in the cervical-dorsal-shoulder area, hip-knee or leg-ankle-foot had a negative and significant impact on the mental dimension of the HRQL (Table 3, Fig. 1). In turn, this component is negatively and significantly correlated with the intensity of the MSP (Spearman's rho $=-0.188$ ), the number of locations with MSP (Spearman's rho $=-0.161$ ) and the score on the RMDQ (Spearman's rho $=-0.294)$ (Table 4).

Table 4. Bivariate relationship between socio-demographic variables, musculoskeletal pain characteristics, functional disability related to lumbar pain and SF-36 (component summary scores)

\begin{tabular}{|c|c|c|c|c|}
\hline \multirow{2}{*}{ Variable } & \multicolumn{2}{|r|}{ PCS } & \multicolumn{2}{|r|}{ MCS } \\
\hline & $n$ & Spearman's rho & $n$ & Spearman's rho \\
\hline \multicolumn{5}{|l|}{ Socio-demographic variables } \\
\hline Age (years) & 922 & $-0.188 * *$ & 922 & -0.041 \\
\hline Years working as shellfish gatherers & 916 & $-0.184 * *$ & 916 & -0.045 \\
\hline VNS, pain $(0-10)$ & 922 & $-0.506 * *$ & 922 & $-0.188 * *$ \\
\hline Number of sites with MSP (0-11) & 923 & $-0.480 * *$ & 923 & $-0.161 * *$ \\
\hline RMDQ scores (0-24) & 923 & $-0.648 * *$ & 923 & $-0.294 * *$ \\
\hline
\end{tabular}

PCS physical component summary, MCS mental component summary, VNS verbal numerical scale, MSP musculoskeletal pain, $R M D Q$ Roland-Morris Disability Questionnaire

*** $p \leq 0.001$, statistical significance

In the multivariable analysis, it can be seen that the variables which have an independent effect in predicting the physical dimension of the HRQL are the intensity of the MSP, musculoskeletal comorbidity, the presence of pain in the hip-knee area, the presence of self-reported rheumatic disorders, the presence of back pain and age (Table 5). On applying the same model for the MCS variable, we find that the variables which have an independent effect to predict the mental dimension are the presence of selfreported depressive syndrome and functional disability due to back pain (Table 5). These variables explain 36 and $22.4 \%$ of the variability in the score for the PCS and MCS, respectively. 
Table 5. Multivariate linear regression analyses for SF-36 physical component summary and mental component summary score

\begin{tabular}{|c|c|c|c|c|}
\hline Variable & $\begin{array}{l}\text { Non-standardised } \\
\text { coefficients }\end{array}$ & SE & $p$ & $95 \%$ CI \\
\hline \multicolumn{5}{|l|}{ PCS } \\
\hline \multicolumn{5}{|l|}{ Included variables } \\
\hline VNS (pain; 0-10) & -0.96 & 0.08 & $\leq 0.001$ & $-1.12 ;-0.80$ \\
\hline Number of sites with MSP (0-11) & -0.77 & 0.15 & $\leq 0.001$ & $-1.06 ;-0.47$ \\
\hline Rheumatic disorders (yes/no) & -2.79 & 0.68 & $\leq 0.001$ & $-4.12 ;-1.46$ \\
\hline Hip/knee pain (yes/no) & -2.26 & 0.60 & $\leq 0.001$ & $-3.44 ;-1.08$ \\
\hline Lower back pain (yes/no) & -1.62 & 0.56 & 0.004 & $-2.72 ;-0.52$ \\
\hline Age (years) & -0.06 & 0.03 & 0.030 & $-0.12 ;-0.01$ \\
\hline \multicolumn{5}{|l|}{ Excluded variables $^{\mathrm{a}}$} \\
\hline \multicolumn{5}{|l|}{ MCS } \\
\hline \multicolumn{5}{|l|}{ Included variables } \\
\hline Depressive syndrome (yes/no) & $-1,043.1 * *$ & 79.43 & $\leq 0.001$ & $-1,198.68 ;-887.32$ \\
\hline RMDQ scores $(0-24)$ & $-42.19 * *$ & 6.13 & $\leq 0.001$ & $-54.21 ;-30.18$ \\
\hline
\end{tabular}

$S E$ standard error, $C I$ confidence interval, PCS physical component summary, MCS mental component summary, VNS verbal numerical scale, $M S P$ musculoskeletal pain, $R M D Q$ Roland-Morris Disability Questionnaire

${ }^{a}$ Excluded variables include years working as shellfish gatherers, neck/shoulders/higher back pain, elbow/wrist/hand pain and leg/ankle/foot pain. The RMDQ variable has not been considered as both the PCS and RMDQ register aspects are associated with functional disability, and so, there is a high colinearity between both variables

${ }^{b}$ Excluded variables include age, geographical areas where shellfish are gathered on foot, rheumatic disorders, neck/shoulders/higher back pain, lower back pain, elbow/wrist/hand pain, hip/knee pain, leg/ankle/foot pain, VNS (pain; 0-10) and number of sites with $\operatorname{MSP}(0-11)$

\section{Discussion}

In this study, carried out with a collective of blue-collar workers, we examined the relationship between HRQL and certain features of MSP, socio-demographic factors, lifestyle factors and comorbidity. It reveals a reduction in HRQL in all of the SF-36 health domains, especially those associated with physical health, as reported by other researchers $[9,10]$.

Six factors, namely, intensity of the pain, presence of pain in the hip-knee and lumbar region, selfreported presence of rheumatic disorders, number of locations with pain, and age, appeared to explain the poor physical health of these workers. The differences found in HRQL can be considered also as clinically relevant according to Norman et al. [16] who establishes a difference of five points in the standard scoring system of SF-36 as a minimally important difference. He stated that in the context of comparing group averages, a medium effect size was five points. Among our findings, we would emphasise the fact that the intensity of MSP is one of the predictor of worse physical health. These results coincide with the findings of various authors [12,17] who draw attention to the importance of this parameter in predicting physical health dimensions.

The specific and substantial impact of MSP on health-related quality of life has already been shown in numerous studies [4-10]. The strength of our study is having analysed the impact of the location and number of locations with MSP (studied in five grouped anatomical areas) on the HRQL. This has allowed us to demonstrate that the presence of pain in the hip-knee area, lumbar pain and the number of locations has a major impact on the PCS. Few studies have taken the location and coexistence of MSP into account. Picavet and Hoeymans [6] examined the HRQL of persons with one or more self-reported musculoskeletal diseases and found that the diseases with the worst HRQL for physical dimensions were osteoarthritis of the knee or hip. They also reported that HRQL deteriorated with an increasing number of musculoskeletal conditions. A study [18] that evaluated quality of life with SF-36 in different musculoskeletal diseases also found that those with knee osteoarthritis had the lowest scores in physical functioning and pain, only behind patients with fibromyalgia. We would highlight a study [19] on the impact of MSP carried out on the Spanish population, which shows that the MSPs that most deteriorate physical health are rheumatoid arthritis, osteoarthritis of the knee and lumbar pain, findings that are very similar to our own. Our findings are also in line with those of other authors who have found that health status as measured by the SF-36 is affected by lumbar pain [5, 9] or musculoskeletal co-morbidity [5, 10] or greater age $[4,5,11,12]$. 
Considering our results and the findings of other authors, it would seem that the worse physical health of these workers is associated with MSP, although our models only explain $36 \%$ of the variance of the PCS. As a result, we consider it reasonable to suppose that there are other factors that worsen physical health, for example, health problems other than MSP that we have not recorded, but whose impact has been studied by other authors [4, 5, 11, 20, 21]. However, it is important to note the study [5] on patients with spine disorders, in which even when recording the co-morbidity of all of the systems, their results only explained $12 \%$ of the variance of the PCS, a much lower percentage than that found in our study. Certain socio-economic aspects, such as a low educational level or being manual workers, as is the case with the workers we studied, have also been associated with a worse HRQL [4, 7, 11, 19].

As regards mental health, these workers have similar values to the general reference population. As in our case, the majority of studies $[4,8-10,19,20]$ coincide in the observation that although MSP affects mental health, it does so to a lesser extent than with physical health. It seems reasonable to have found that self-reported depressive syndrome predicts worse mental health, as the SF-36 questionnaire in its MH dimension includes questions on these symptoms. Bair et al. [22] in a study of patients with chronic MSP found that the presence of depression especially affected several dimensions (SF, VT and GH) that contribute to the score on the MCS. In turn, our results coincide with the study of Hurwitz et al. [23] which shows that functional disability due to back pain is a risk factor for the mental health dimension on the SF-36. We consider that the fact that these workers have values close to the reference population may have limited the predictive value of the variables studied ( $22 \%$ of the variance of the MCS). Another possibility is the fact that as in the case of physical health, we have not studied determining variables of mental health, such as psychosocial factors associated with the occupation [24] or cognitive aspects associated with pain $[12,17]$.

Several limitations of the present study are worthy of mention. First, the participants were volunteers, so this bias in the selection may have had some influence on the results. The quality of the data is based on the eventuality that the wish to take part is not associated with the participant's state of health or other variables of interest included in the study. However, the bias of the non-participants may have led to overestimation (due to a greater participation of persons with pain) or underestimation (due to the "healthy volunteer" effect) of the score on the HRQL. Some authors [25] have found that persons with pain or worse health may be encouraged to take part in studies of this kind. Nevertheless, it should be noted that the socio-demographic characteristics of the non-participants are similar to those of the sample in terms of sex and age, and as a result of which, it would be expected that the co-morbidity of the groups is comparable. Secondly, neither the physical activity nor the co-morbidity has been collected with validated questionnaires which might compromise the relative results of these variables. Finally, a potential limitation is the fact that the design used in this study is cross-sectional; therefore, the causality of the associations cannot be established from the findings.

\section{Conclusions}

The results of this study have confirmed the major impact of musculoskeletal pain on health-related quality of life, especially in areas associated with physical health. The intensity of the pain, pain in the hip and knee, lower back pain, functional disability due to back pain as well as the number of locations of MSP were found to have a clearly detrimental impact on the quality of life of the affected workers. In spite of limitations due to the cross-sectional study design, these results suggest to which features of musculoskeletal pain we should give preference in order to increase the HRQL among fishing sector workers. The application of possible therapeutic and/or preventive strategies in these workers should take these features into consideration.

\section{Acknowledgments}

We would like to thank the Xunta de Galicia (Regional Ministry of Maritime Affairs and the Regional Ministry of Labour), the Shellfish Gatherers' Associations of Galicia, and the Faculty of Physiotherapy of the University of A Coruña for supporting and promoting this study. We would also like to thank Mr. Lino Lema for setting up the project "Protect your body, it's your instrument of work. Prevention of musculoskeletal problems in shellfish gatherers", which served as the basis for gathering the data for this study, and all of the fishing extension agents for their collaboration in organising the preventive physiotherapy workshops. Finally, we would express our most sincere gratitude to all of the shellfish gatherers who voluntarily took part in this study. 


\section{Conflict of interest}

B Rodríguez-Romero, S Pita-Fernández and S Pértega-Díaz declare that they have no conflict of interest.

\section{References}

1. Nicholas MK (2008) Pain management in musculoskeletal conditions. Best Pract Res Clin Rheumatol 22:451-470

2. Bombardier C (2000) Outcome assessments in the evaluation of treatment of spinal disorders: summary and general recommendations. Spine (Phila Pa 1976) 25:3100-3103

3. Rodríguez-Romero B, Pita-Fernandez S, Raposo-Vidal I, Seoane-Pillado T (2012) Prevalence, co-occurrence, and predictive factors for musculoskeletal pain among shellfish gatherers. Clin Rheumatol 31:283-292

4. Sprangers MA, de Regt EB, Andries F et al (2000) Which chronic conditions are associated with better or poorer quality of life? J Clin Epidemiol 53:895-907

5. Fanuele JC, Birkmeyer NJ, Abdu WA, Tosteson TD, Weinstein JN (2000) The impact of spinal problems on the health status of patients: have we underestimated the effect? Spine (Phila Pa 1976) 25:1509-1514

6. Picavet HS, Hoeymans N (2004) Health related quality of life in multiple musculoskeletal diseases: SF-36 and EQ5D in the DMC3 study. Ann Rheum Dis 63:723-729

7. Bingefors K, Isacson D (2004) Epidemiology, co-morbidity, and impact on health-related quality of life of selfreported headache and musculoskeletal pain - a gender perspective. Eur J Pain 8:435-450

8. van der Waal JM, Terwee CB, van der Windt DA, Bouter LM, Dekker J (2005) Health-related and overall quality of life of patients with chronic hip and knee complaints in general practice. Qual Life Res 14:795-803

9. Morken T, Riise T, Moen B et al (2002) Frequent musculoskeletal symptoms and reduced health-related quality of life among industrial workers. Occup Med (Lond) 52:91-98

10. Saastamoinen P, Leino-Arjas P, Laaksonen M, Martikainen P, Lahelma E (2006) Pain and health related functioning among employees. J Epidemiol Community Health 60:793-798

11. Salaffi F, De Angelis R, Stancati A, Grassi W, MArche Pain, Prevalence INvestigation Group (MAPPING) study (2005) Health-related quality of life in multiple musculoskeletal conditions: a cross-sectional population based epidemiological study. II. The MAPPING study. Clin Exp Rheumatol 23:829-839

12. Severeijns R, van den Hout MA, Vlaeyen JW, Picavet HS (2002) Pain catastrophizing and general health status in a large Dutch community sample. Pain 99:367-376

13. Ware JE Jr (2000) SF-36 health survey update. Spine 25:3130-3139

14. Alonso J, Regidor E, Barrio G, Prieto L, Rodriguez C, de la Fuente L (1998) Population reference values of the Spanish version of the Health Questionnaire SF-36. Med Clin (Barc) 111:410-416

15. Picavet HS, Schouten JS (2003) Musculoskeletal pain in the Netherlands: prevalences, consequences and risk groups, the DMC(3)-study. Pain 102:167-178

16. Norman GR, Sloan JA, Wyrwich KW (2003) Interpretation of changes in health-related quality of life: the remarkable universality of half a standard deviation. Med Care 41:582-592

17. Dysvik E, Lindstrom TC, Eikeland OJ, Natvig GK (2004) Health-related quality of life and pain beliefs among people suffering from chronic pain. Pain Manag Nurs 5:66-74

18. Yilmaz F, Sahin F, Ergoz E et al (2008) Quality of life assessments with SF 36 in different musculoskeletal diseases. Clin Rheumatol 27:327-332

19. Carmona L, Ballina J, Gabriel R, Laffon A, EPISER Study Group (2001) The burden of musculoskeletal diseases in the general population of Spain: results from a national survey. Ann Rheum Dis 60:1040-1045

20. Alonso J, Ferrer M, Gandek B et al (2004) Health-related quality of life associated with chronic conditions in eight countries: results from the International Quality of Life Assessment (IQOLA) Project. Qual Life Res 13:283-298

21. Fortin M, Lapointe L, Hudon C, Vanasse A, Ntetu AL, Maltais D (2004) Multimorbidity and quality of life in primary care: a systematic review. Health Qual Life Outcomes 2:51

22. Bair MJ, Wu J, Damush TM, Sutherland JM, Kroenke K (2008) Association of depression and anxiety alone and in combination with chronic musculoskeletal pain in primary care patients. Psychosom Med 70:890-897

23. Hurwitz EL, Morgenstern H, Yu F (2003) Cross-sectional and longitudinal associations of low-back pain and related disability with psychological distress among patients enrolled in the UCLA Low-Back Pain Study. J Clin Epidemiol 56:463-471

24. Cheng Y, Kawachi I, Coakley EH, Schwartz J, Colditz G (2000) Association between psychosocial work characteristics and health functioning in American women: prospective study. BMJ 320:1432-1436

25. Picavet HS, Hazes JM (2003) Prevalence of self reported musculoskeletal diseases is high. Ann Rheum Dis 62:644-650 